\title{
A REMARK ON A NORM INEQUALITY FOR SQUARE MATRICES
}

M. D. MARCUS

In a recent paper $W$. Gautschi [1] obtained the following inequality for a non-nilpotent $n$-square matrix $A$ :

$$
\left(\sum_{j=1}^{n}\left|\lambda_{i}\right|^{2 p}\right)^{1 / 2} \leqq\left\|A^{p}\right\| \leqq c_{0} p^{k-1}\left(\sum_{j=1}^{n}\left|\lambda_{j}\right|^{2 p}\right)^{1 / 2}
$$

where $\|A\|^{2}=\sum_{i, j=1}^{n}\left|a_{i j}\right|^{2}, p$ is an integer, $c_{0}$ a constant depending only on $A$, and $k$ the maximum multiplicity of any characteristic root of $A$. By a reduction to Jordan canonical form an inequality of type (1) may be obtained for any convergent matrix power series $g(A), g(z)=\sum_{n=0}^{\infty} a_{n} z^{n}, a_{n}$ real, which in the case $g(z)=z^{p}$ implies (1).

TheOREM. Let $g(z)$ have radius of convergence $\rho>0$. Let $A$ be an $n$-square matrix. whose characteristic roots satisfy $\left|\lambda_{i}\right|<\rho$. Let the multiplicity of $\lambda_{i}$ be $k_{i}, i=1, \cdots, \alpha$, and $k=\max k_{i}$. Then if $A$ is not nilpotent there exists a constant $c$ depending only on $A$ such that

$$
\left(\sum_{j=1}^{n}\left|g\left(\lambda_{j}\right)\right|^{2}\right)^{1 / 2} \leqq\|g(A)\| \leqq c\left(\sum_{j=1}^{\alpha} \sum_{s=0}^{k_{j}-1} \frac{\left(k_{j}-s\right)}{(s !)^{2}}\left|g^{(s)}\left(\lambda_{j}\right)\right|^{2}\right)^{1 / 2} .
$$

Proof. A very slight extension of Gautschi's argument yields the lower bound. For by Schur's theorem [2] there exists a unitary matrix $U$ reducing $A$ to triangular form $D$ with characteristic roots along the main diagonal. Then

$$
U A U^{*}=D \text { and } U U^{*}=I
$$

imply

$$
\begin{aligned}
\|g(A)\|^{2} & =\operatorname{tr}\left(g(A) g^{*}(A)\right)=\operatorname{tr}\left(g(A) g\left(A^{*}\right)\right) \\
& =\operatorname{tr}\left(U g(A) g\left(A^{*}\right) U^{*}\right)=\operatorname{tr}\left(U g(A) U^{*} U g\left(A^{*}\right) U^{*}\right) \\
& =\operatorname{tr}\left(g\left(U A U^{*}\right) g\left(U A^{*} U^{*}\right)\right)=\operatorname{tr}\left(g(D) g\left(D^{*}\right)\right) \\
& =\operatorname{tr}\left(g(D) g^{*}(D)\right) \geqq \sum_{j=1}^{n}\left|g\left(\lambda_{j}\right)\right|^{2}
\end{aligned}
$$

since $g(D)$ is a triangular matrix with $g\left(\lambda_{j}\right)$ along the main diagonal. To obtain the upper bound let $T$ be such a nonsingular matrix that $T A T^{-1}=\operatorname{diag}\left(E_{1}, \cdots, E_{\alpha}\right)=\operatorname{diag} E_{j} ; E_{j}=\lambda_{j} I_{j}+U_{j}, I_{j}$ the $k_{j}$-square

Received by the editors April 17, 1954. 
identity matrix and $U_{j}$ the $k_{j}$-square matrix with 1 along the superdiagonal and 0 elsewhere. Then setting $c=\|T\|\left\|T^{-1}\right\|$, we have

$$
\begin{aligned}
\|g(A)\| & =\left\|T^{-1} T g(A) T^{-1} T\right\|=\left\|T^{-1} g\left(T A T^{-1}\right) T\right\| \leqq c\left\|g\left(\operatorname{diag} E_{j}\right)\right\| \\
& =c\left\|\operatorname{diag} g\left(E_{j}\right)\right\| .
\end{aligned}
$$

An elementary calculation shows (S. Lefschetz [3])

$$
g\left(E_{j}\right)=g\left(\lambda_{j} I_{j}+U_{j}\right)=\left(\begin{array}{cccc}
g\left(\lambda_{j}\right) & g^{(1)}\left(\lambda_{j}\right) \cdots & g^{\left(k_{j}-1\right)}\left(\lambda_{j}\right) /\left(k_{j}-1\right) ! \\
\vdots & \vdots & \vdots & \vdots \\
\vdots & \vdots & \vdots & g^{(1)}\left(\lambda_{j}\right) \\
0 & \vdots & : & g\left(\lambda_{j}\right)
\end{array}\right)
$$

and hence by summing down the diagonals,

$$
\left\|g\left(E_{j}\right)\right\|^{2}=\sum_{s=0}^{k_{j}-1}\left(\left(k_{i}-s\right) /(s !)^{2}\right)\left|g^{(s)}\left(\lambda_{j}\right)\right|^{2}
$$

and

$$
\|g(A)\|^{2}=c^{2} \sum_{j=1}^{\alpha}\left\|g\left(E_{j}\right)\right\|^{2} \leqq c^{2} \sum_{j=1}^{\alpha} \sum_{s=0}^{k_{j}-1} \frac{\left(k_{j}-s\right)}{(s !)^{2}}\left|g^{(s)}\left(\lambda_{j}\right)\right|^{2},
$$

and the proof is complete.

If $g(z)=z^{p}$ we may easily estimate $\left\|g\left(E_{j}\right)\right\|$. We distinguish two possibilities: (i) $p>k_{j}-1$, (ii) $p \leqq k_{j}-1$. If (i), $\lambda_{j}=0$ implies $\left\|g\left(E_{j}\right)\right\|=0$, and $\lambda_{j} \neq 0$ implies

$$
\left\|g\left(E_{j}\right)\right\|^{2}=\left|\lambda_{j}\right|{ }^{2 p} \sum_{i=0}^{k_{j}-1}\left(\begin{array}{l}
p \\
s
\end{array}\right)^{2}\left(k_{j}-s\right)\left|\lambda_{j}\right|^{-2 s} \leqq c_{1}\left|\lambda_{j}\right|{ }^{2 p} p^{2 k-2} .
$$

If (ii), $\lambda_{j}=0$ implies $\left\|g\left(E_{j}\right)\right\|^{2}=\left(k_{j}-p\right)(p !)^{2} \leqq c_{2} p^{2 k-2}$, and again $\lambda_{j} \neq 0$ implies the same conclusion as in (i), where $c_{1}$ and $c_{2}$ depend only on the multiplicities and magnitudes of the $\lambda_{j}$. Thus

$$
\|g(A)\|^{2} \leqq c^{2} p^{2 k-2}\left(c_{2}+c_{1} \sum_{j=1}^{n}\left|\lambda_{j}\right|^{2 p}\right) \leqq c_{0}^{2} p^{2 k-2} \sum_{j=1}^{n}\left|\lambda_{i}\right|^{2 p}
$$

since $A$ not nilpotent is equivalent to $\sum_{j=1}^{n}\left|\lambda_{j}\right|^{2} \neq 0$. Noting that $\sum_{j=1}^{n}\left|g\left(\lambda_{j}\right)\right|^{2}=\sum_{j=1}^{n}\left|\lambda_{j}\right|^{2 p}$, Gautschi's result follows. We may employ the inequality to unify conveniently the familiar results concerning the asymptotic behavior of the system of ordinary differential equations $\dot{x}=A x, A$ an $n$-square constant matrix, $x$ an $n$-column vector, with fundamental matrix of solutions exp $(t A)$. Consider $g(z)=\exp (t z), g^{(s)}(z)=t^{*} \exp (t z), t \geqq 0$, and the resulting inequality 


$$
\begin{aligned}
\left(\sum_{j=1}^{n}\left|\exp \left(t \lambda_{j}\right)\right|^{2}\right)^{1 / 2} & \leqq\|\exp (t A)\| \\
& \leqq c\left(\sum_{j=1}^{\alpha} \sum_{i=0}^{k_{j}-1} \frac{\left(k_{j}-s\right)}{(s !)^{2}}\left|\left(t^{\circ} \exp \left(t \lambda_{j}\right)\right)\right|^{2}\right)^{1 / 2} .
\end{aligned}
$$

Letting $r_{j}=\operatorname{Re}\left(\lambda_{j}\right)$ we have

$$
\begin{aligned}
\left(\sum_{j=1}^{n} \exp \left(2 r_{j} t\right)\right)^{1 / 2} & \leqq\|\exp (t A)\| \\
& \leqq c\left(\sum_{j=1}^{\alpha} \sum_{s=0}^{k j-1} \frac{\left(k_{j}-s\right)}{(s !)^{2}} t^{2 \triangleleft} \exp \left(2 t r_{j}\right)\right)^{1 / 2}
\end{aligned}
$$

and we conclude immediately that:

(i) $r_{j}>0$ for some $j$ implies $\lim _{t \rightarrow \infty}\|\exp (t A)\|=\infty$,

(ii) $r_{j}<0$ for all $j$ implies $\lim _{t \rightarrow \infty}\|\exp (t A)\|=0$,

(iii) $r_{j} \leqq 0$ for all $j$ and $r_{j}=0$ only if $k_{j}=1$ implies $\|\exp (t A)\|$ is bounded on $[0, \infty)$.

In case $A$ is nilpotent, $\exp (t A)$ has as entries polynomials in $t$.

\section{REFERENCES}

1. Werner Gautschi, The asymptotic behavior of powers of matrices, Duke Math. J. vol. 20 (1953) pp. 127-140.

2. I. Schur, Uber die charakteristischen Wurzeln einer linearen Substitution mit einer Anwendung auf die Theorie der Integralgleichungen, Math. Ann. vol. 66 (1909) pp. 488-510.

3. S. Lefschetz, Lectures on differential equations, Annals of Mathematics Studies, no. 14, p. 6.

The University of British Columbia 